\title{
FABRICATION AND CHARACTERIZATION OF Ni-CNT COMPOSITES BY ELECTRICAL EXPLOSION OF WIRE IN DIFFERENT LIQUIDS
}

\begin{abstract}
In this study, Ni-CNT powders and colloids were synthesized via the Electrical explosion of wire (EEW) in different liquid conditions. The influence of ambient solvents (D.I. Water, ethanol, methanol, acetone and ethylene-glycol) on characteristics of the as-synthesized Ni-CNT was investigated. The morphology and size were observed by field emission scanning electron microscopy (FE-SEM). The Ni particles were spherical or near spherical shape. The phase of the composite powders analyzed via X-ray diffraction demonstrate the presence of CNTs in composite powders is not affect the structure of Ni. However, the phase of the composites was changed based on the changing of liquid conditions. Stability of colloids was investigated by Turbiscan technique. Magnetic properties were also investigated by Vibrating sample magnetometer (VSM) at room temperature. The as-synthesized composite powders revealed a ferromagnetic characteristic material.

Keywords: Nickel-Carbon nanotubes composite, Carbon nanotubes, Electrical explosion of wire, Nano-colloid, Magnetic properties
\end{abstract}

\section{Introduction}

Carbon nanotubes are the stiffest and strongest known fibers and have unique electrical properties [1-3]. Many researchers treat carbon nanotubes as one-dimensional material templates [4]. Owing to the outstanding mechanical, thermal and electrical properties, accompanied by the low density, CNTs become an excellent candidate for many applications as additives in polymers, catalysts, electromagnetic-wave absorption and shielding, sensor. Nickel is a good ferromagnetic material, which can be deposited in a layer of carbon nanotubes to obtain one-dimensional magnetic materials and further to obtain one-dimensional nano-materials in the application of magnetic recording. In some recent studies, nickel has been selected as the matrix to synthesized Ni-CNT composite with the reinforcement element was CNT. The Ni-CNT composites are usually fabricated by coating in order to make a thin film, via powder metallurgy (PM) process or mixing process and then combine with sintering to obtain the bulk samples. The aim of these processes is offering the possibility of obtaining uniform parts and reducing production costs [5-8]. Up to now, many authors have attempted to develop new ways for synthesizing Ni-CNT nanocomposites due to their excellent enhanced not only mechanical properties, but also thermal properties, electrical conductivity and magnetic properties.

The electrical explosion of wire (EEW) has become an attractive method for fabrication of the nano colloid and nano- sized powders due to the simple and promising approach for low-cost production [9-13]. In this study, the Ni-CNT colloid and Ni-CNT composite powder were prepared by the EEW in different conditions, including ethanol, acetone, deionized water, ethylene glycol, and methanol. According to the literature, the ambient environment is an important parameter that affects not only the size, shape and the distribution of the particle, but also the structure of the powder [9,10,14]. Therefore, the influences of the ambient medium on the properties of the as-synthesized Ni-CNT composite powders were investigated in this work. The stability of the fluids, structural and magnetic properties of the as-synthesized composite powders were also characterized.

\section{Experimental}

Carbon nanotubes were first mixed well with the liquid by stirring in ethanol, acetone, deionized water, ethylene glycol, and methanol, respectively for 2 hours to obtain the CNTs suspension. Then Ni wire with diameter $0.25 \mathrm{~mm}$ was exploded in the CNTs suspension. The experimental setup of the EEW process has been described previously $[15,16]$. A high-density current pulse passes via the wire, which is heated due to Joule heating, vaporized, turned into plasma state, yielding a shockwave which was scattered in the ambient media. After exploding, the stability of the fluids was measured via a Turbiscan LAB equipment. 
The aforementioned instrumentation can detect concentration variations in the prepared colloids by scanning the entire height of the sample via transmission and light backscattering. A small amount of the powders was isolated from the suspension via centrifugation and dried under vacuum for 5 hours for further analyses via XRD and FE-SEM. Magnetic properties the Ni-CNT composite powder was investigated in the room temperature by using a vibrating sample magnetometer (VSM).

\section{Result and discussion}

Figure 1 shows the FE-SEM images of CNTs, Ni powder and Ni-CNTs powders. It denotes that the morphology and structure of the CNTs as characterized in literature [17-19]. The Ni powder was spherical or near spherical in shape. During the EEW process with the CNTs dispersion, Ni powders just generated in the explosion zone could react with CNTs and bond together and then diffuse into the solvent. Under the effect of explosion phenomenon, the liquid ambient was always stirred resulting in homogenous distribution of the Ni-CNTs in the obtained colloid. The morphology results illustrate that the CNTs were separated, distributed and bonded to the surface of the Ni particles [8].

The particle size distribution of Ni-CNT composite powders is shown in the Fig. 2 . The mean size value $\left(D_{50}\right)$ and the diameter range are listed in the Table 1. It illustrates that Ni-CNT powder exploded in DI water condition has a smaller diameter range of the distribution size $(<25 \mu \mathrm{m})$ and the smallest value of the mean size $\left(\mathrm{D}_{50}=5.61 \mu \mathrm{m}\right)$. In the other conditions the distribution range is broader $(<60 \mu \mathrm{m})$ and $\mathrm{D}_{50}$ values are slightly different $17.18 \mu \mathrm{m}, 18.86 \mu \mathrm{m}, 20.70 \mu \mathrm{m}$ and $21.71 \mu \mathrm{m}$ (Tab. 1). For all samples the average size of the composite powders is under 20
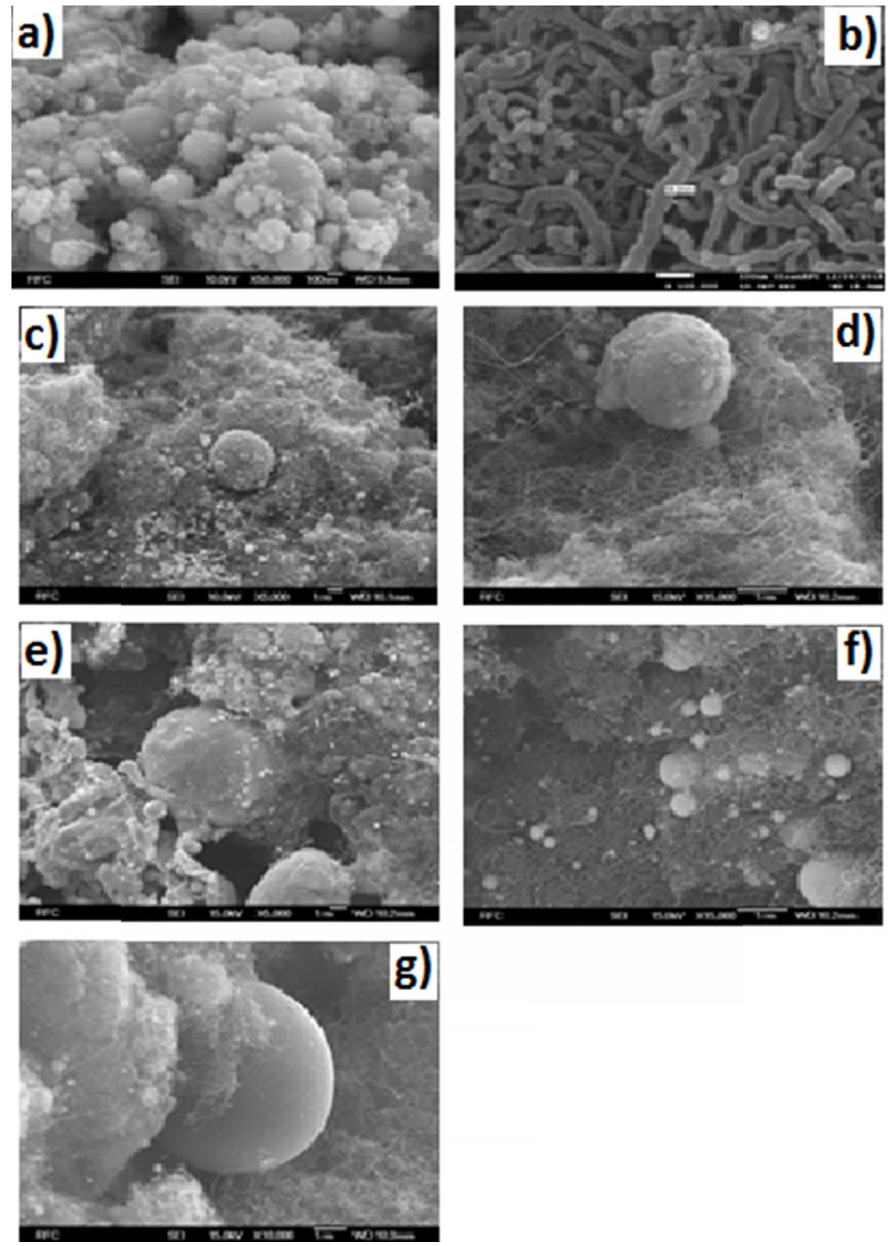

Fig. 1. FE-SEM of a) Ni powder, b) CNTs and Ni-CNT composite powders produced via EEW in c) ethanol, d) acetone, d) DI water, f) EG, and g) methanol
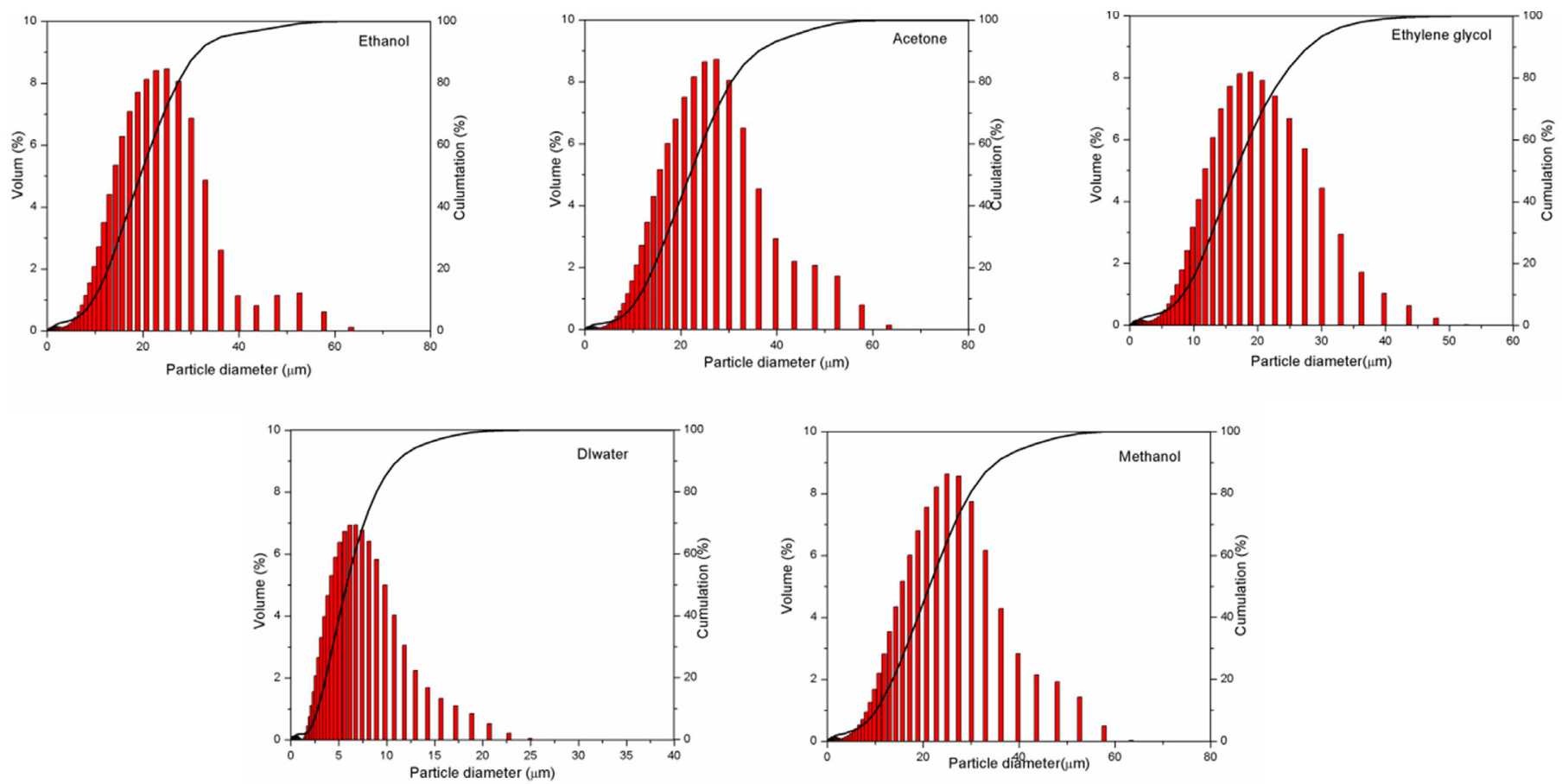

Fig. 2. The particle size distribution of the as-prepared Ni-CNT composite powder 
$\mu \mathrm{m}$. However, as observed from the FE-SEM image, the size of Ni particles are mostly under $100 \mathrm{~nm}$. The difference here could be explained by the combination of Ni nanoparticles and CNTs due to the tendency of agglomeration of them. In DI water and EG medium conditions the $\mathrm{D}_{50}$ values are quite small illustrates that the agglomeration of the Ni-CNT powder in these conditions is reduced in comparison to the other conditions. It predicts that the Ni-CNT fluids in DI water and EG condition could be more homogenous and stable than in others.

TABLE 1

The mean size values and distribution range

\begin{tabular}{|c|c|c|}
\hline \hline Conditions & Mean size, $\mathbf{D}_{\mathbf{5 0}}(\boldsymbol{\mu m})$ & Diameter range \\
\hline DI water & 5.61 & $<25 \mu \mathrm{m}$ \\
\hline Ethylene glycol & 17.18 & $<50 \mu \mathrm{m}$ \\
\hline Ethanol & 18.86 & $<60 \mu \mathrm{m}$ \\
\hline Methanol & 20.70 & $<60 \mu \mathrm{m}$ \\
\hline Acetone & 21.71 & $<60 \mu \mathrm{m}$ \\
\hline
\end{tabular}

To observe the stability of Ni-CNT fluids, Turbiscan method was used and the measurement was carried out in 24 hours. Fig. 3 shows the transmission and the backscatter-

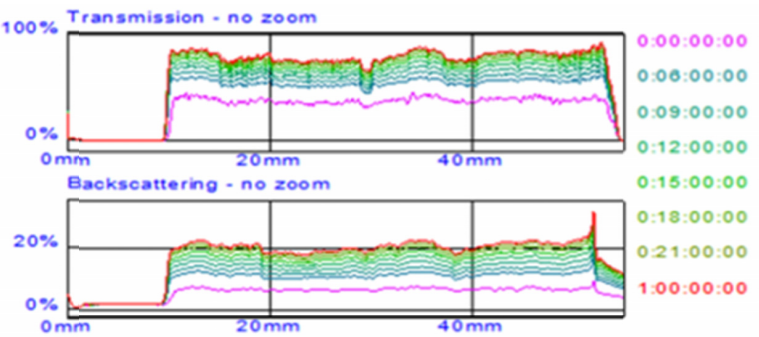

a) Ethanol

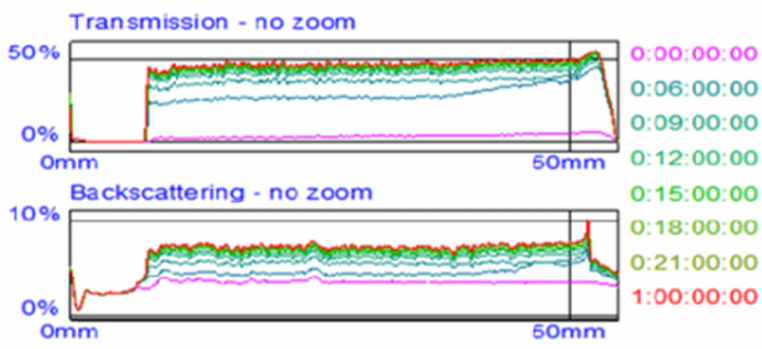

c) DI water ing profile of the fluids. It can be seen that both these signals increased over the time. From observation in transmission profile, the variation gradually rose at the top of the bottle. The sedimentation of Ni-CNT powder happened, leading to the reducing of the variation of the concentration of the dispersed phase between the top part and the bottom part. Therefore, it caused the transmission signal increase. When almost all particles deposited at the bottom, the transmission signal became flat without phase thickness. Among the samples, only sample with EG condition did not show the transmission profile at the first stage of analyzing (Fig. 3d). that means no transmission light went through the bottle due to opaque Ni-CNT fluid. It illustrates the dispersion of the Ni-CNT in EG is more homogenous than in other condition. For the Ni-CNT fluid sample in acetone medium, the composite powder settled down very fast so the change in transmission signal is not separated over the time (Fig. 3b). Generally, the sedimentation rate of Ni-CNT composite powders increase in order of EG, ethanol, DI water and methanol, respectively. After 24 hours, both of the variation of transmission and backscattering signal soared and then reached constant. At that time, almost all Ni-CNT particles settled down, therefore, the variation was constant due to only pure ambient condition. This behavior revealed that Ni-CNT

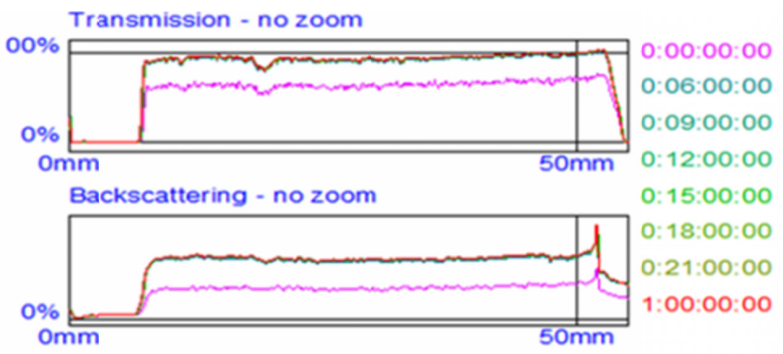

b) Acetone

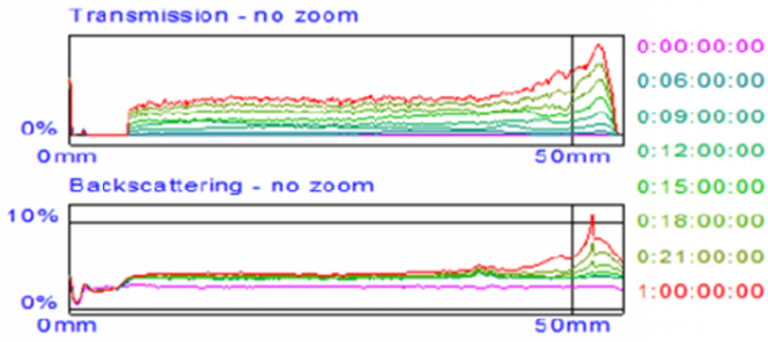

d) Ethylene glycol

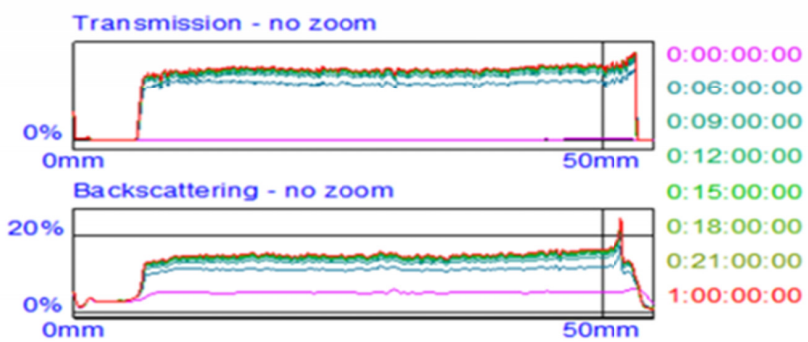

e) Methanol

Fig. 3. Transmission and backscattering profile of Ni-CNT fluids prepared by EEW in different medium conditions in 24 hours 
fluids prepared by EEW were unstable in these liquid conditions. The unstable Ni-CNT fluids could be caused by the high agglomerate tendency of CNTs and between Ni particles and CNTs leads to the formation of clusters or bundles, and resulting in the settle down of the Ni-CNT in the fluids. Furthermore, the difference of the density and viscosity of liquid conditions (Table 2) could affect the behavior of the fluids. Due to the low of density and viscosity of acetone and methanol, the agglomeration of Ni-CNT occurred at the beginning then it settles down very quickly during testing time, resulting in the separation of the signal evidently. Ethelene glycol shows a highest density and viscosity value leading to the lowest sedimentation rate of Ni-CNT powder in comparison with the others. The density of DI water is higher, however, its viscosity is lower than ethanol, therefore, the transmission and backscattering signal in case of ethanol condition were dense and the sedimentation was slower than in DI water.

TABLE 2

The density value and viscosity value of liquid conditions (at room temperature)

\begin{tabular}{|c|c|c|}
\hline \hline Liquid condition & Density $\mathbf{( g / \mathbf { c m } ^ { \mathbf { 3 } } )}$ & Viscosity (mPa.s) \\
\hline Ethylene glycol & 1.113 & 19.83 \\
\hline DI water & 1 & 0.89 \\
\hline Ethanol & 0.7893 & 1.2 \\
\hline Methanol & 0.792 & 0.545 \\
\hline Acetone & 0.7845 & 0.92 \\
\hline
\end{tabular}

The XRD patterns of Ni-CNT composite powders are shown in Fig. 4. In all samples, the appearing of a small peak at $2 \theta \sim 26^{\circ}$ corresponding to CNTs, it illustrates to the presence of CNTs in the Ni-CNT composite powders. Generally, the XRD patterns of $\mathrm{Ni}$ in composite powders are not the same demon-

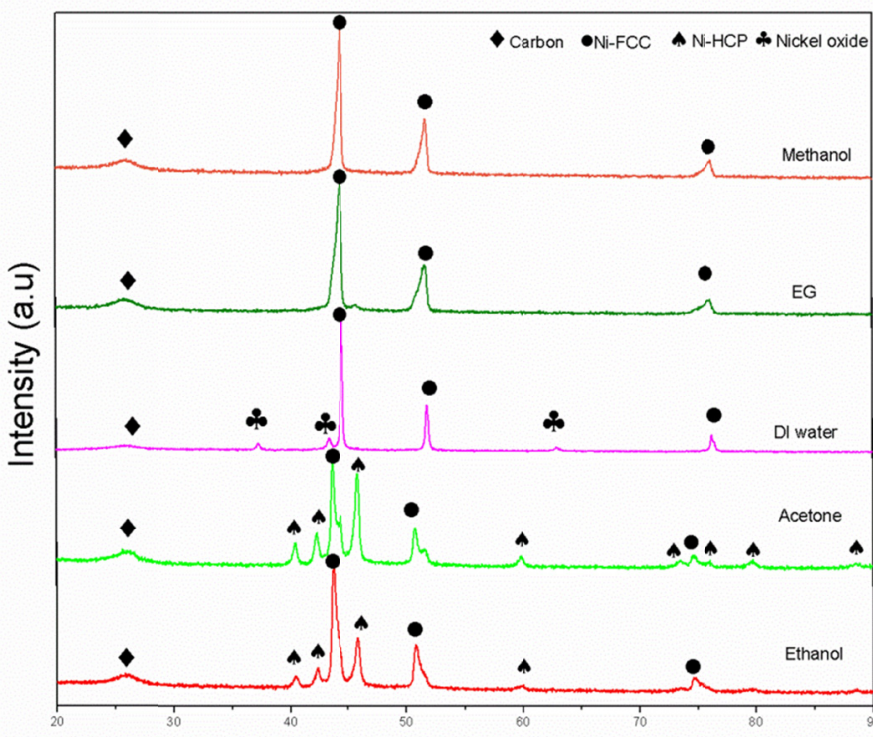

$2 \theta$ (degree)

Fig. 4. XRD patterns of Ni-CNT composite powders produced via EEW in different liquid conditions strates that the presence of CNTs did not affect the structure of $\mathrm{Ni}$, but the changing in phase structure of $\mathrm{Ni}$ is caused by ambient solution.

The XRD patterns of the Ni-CNT composite powders exploded in EG and Methanol show peaks of pure Ni (Ni-FCC) and small peak of CNTs only. In DI water condition, a mixed phase of pure $\mathrm{Ni}$ and Nickel oxide $(\mathrm{NiO})$ were observed. With acetone and ethanol solvents, the patterns of Ni-CNT powder were totally different. Both of the Ni-FCC and Ni-HCP structure could be observed with their peaks match with JCPDF card 040850 and JCPDF card 45-1027, respectively. In fact, Ni-HCP is not a thermodynamically stable crystal structure of $\mathrm{Ni}$, and only observed under the high levels of stress or strain conditions, like those during ion bombardment [20]. In comparasion with EEW process, Ni powder was exploded in explosion-plasma zone, so it leads to the formation of HCP structure. However, the Ni-HCP structure only occurred in organic solvents [13] illustrates that the organic liquid affected Ni structure more strongly. Nevertheless, most of the peaks could not be assigned due to carbon and oxygen atoms in the solvent are thought to have been integrated the Ni crystal structure into metastable phase $[9,10]$.

Magnetic properties of as-synthesized Ni-CNT composite powders were investigated at room temperature by using a VSM with an applied field $-20,000 \mathrm{kOe} \leq \mathrm{H} \leq 20,000 \mathrm{kOe}$. Figure 5 shows the magnetization of samples. The value of saturation magnetization and coercive force were shown in (Table 3 ). These values of the Ni-CNT powders prepared in DI water, Ethylene glycol, and Methanol are absolutely higher than those in the rest medium conditions. This phenomenon can be explained based on the difference in the structure of the Ni-CNT powders. With DI water, Ethylene glycol, and Methanol, the phase of Ni were pure $\mathrm{Ni}$ or $\mathrm{Ni}$ and $\mathrm{Ni}$ oxide with a higher magnetic properties, meanwhile the metastable phase in Ethanol and Acetone condition has a lower magnetic properties.

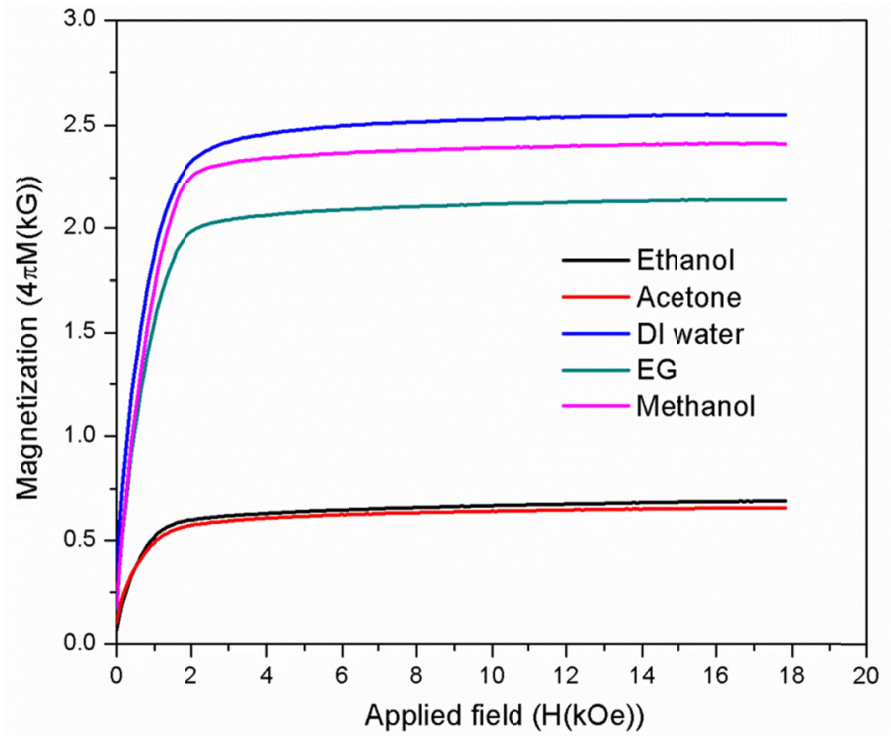

Fig. 5. Magnetization of the Ni-CNT powders measured at room temperature a) Ethanol, b) Acetone, c) DI water, d) Ethylene glycol e) Methanol 


\section{RERERENCES}

Saturation magnetization and coercivity

\begin{tabular}{|c|c|c|}
\hline \hline Conditions & Coercivity (kOe) & $\begin{array}{c}\text { Magnetization value } \\
(\mathbf{4} \boldsymbol{\pi M}(\mathbf{k G}))\end{array}$ \\
\hline Acetone & 0.17167 & 0.65 \\
\hline Ethanol & 0.14039 & 0.68 \\
\hline Ethylene glycol & 0.3608 & 2.13559 \\
\hline Methanol & 0.37146 & 2.4111 \\
\hline DI water & 0.75911 & 2.54925 \\
\hline
\end{tabular}

\section{Conclusions}

The Ni-CNT composite powders were prepared via EEW in different liquid conditions successfully, and its properties depend on the ambient medium of EEW process but not the present of CNTs.

The powder produced in Methanol and EG only show phases of pure Ni. While in ID water, mixed phases of pure $\mathrm{Ni}$ and $\mathrm{Ni}$ oxide was observed. In cases of ethanol, acetone the phases were changed to metastable phases. The average saturation magnetization of powders prepared in DI water, methanol and EG are higher than those in ethanol and acetone. The NiCNT nanofluids prepared by EEW in ethanol, acetone, DI water, ethylene glycol, and methanol are unstable colloids. However, DI water is a good condition to prepare Ni-CNT composite powder with a small particle size $\left(\mathrm{D}_{50} \sim 5.61 \mu \mathrm{m}\right)$ and high magnetization value $(2.549 \mathrm{kG})$. Methanol and EG medium are the best conditions for preventing the oxidation.

\section{Acknowledgements}

This work was financially supported by the 2015 Research Fund of the University of Ulsan.
[1] S.R. Bakshi, D.L.A. Agarwal, International Materials Reviews A55, 41 (2010).

[2] D. Qian, G.J.W, W.K. Liu, American Society of Machanical Engineers 55, (2002)

[3] S. Iijima, Nature 354, 56 (1991).

[4] M.A. Correa-Duarte, L.M.L.-M, Journal of Materials Chemistry 16, 22 (2006).

[5] S. Suarez, F.L.F. Mucklich, Materials Science and Engineering a Structural Materials Properties Microstructure and Processing 587, 381 (2013).

[6] H. Wang, Z.H.Z., Z.Y. Hu, F.C. Wang, S.L. Li, E. Korznikov, X.C. Zhao, Y. Liu, Z.F. Liu, Z. Kang, Scientific Reports 6, (2016).

[7] S. Yamanaka, R.G, A. Kawasaki, H. Sakamoto, Y. Mekuchi, M. Kun, T. Tsukada, Materials Transactions 48, 2506 (2007).

[8] Thuyet-Nguyen Minh, H.-N.H., Won Joo Kim, Ho Yoon Kim, Jin-Chun Kim, Journal of Korean Powder Metallurgy Institute 23, 213 (2016).

[9] C. Cho, Y.C.H, C. Kang, Y.S. Jin, G H. Rim, Journal of the Korean Physical Society 57, 1807 (2010).

[10] L.H. Bac, B.K. Kim, J.S. Kim, J.C. Kim, Journal of Magnetics 16, 435 (2011).

[11] L.H. Bac, J.S. Kim, J.C. Kim, C.K. Rhee, Journal of Korean Powder Metallurgy Institute 36, 795 (2010).

[12] W.H. Jiang, K.Y, Ieee Transactions on Plasma Science 26, 1498 (1998).

[13] Y.A. Kotov, O.M.S, Nanostructured Materials 12, 119 (1999).

[14] L.H. Bac, G.S. Y, J.S. Kim, J.C. Kim, C.K. Rhee,Modern Physics Letters B 23, 3897 (2009).

[15] L.H. Bac, J.S. Kim, J.C. Kim, Research on Chemical Intermediates 36, 795 (2010).

[16] L.H. Bac, Y.S.K, J.S. Kim, Y.I. Lee, D.W. Lee, J.C. Kim, Materials Research Bulletin 45, 352 (2010).

[17] H.W. Kroto, J.R.H., S.C. Obrien, R.F. Curl, R.E. Smalley, Nature 318, 162 (1985).

[18] D. Ugarte, Nature 359, 707 (1992).

[19] M. Zeiger, N.J, M. Asian, D. Weingarth, V. Presser, Carbon 84, 584 (2015).

[20] E. Johnson, T.W.a.W.A.G. Phase Transitions 1, 23 (1979). 